\title{
IMPAIRED NEUROPSYCHOLOGICAL PROFILE IN HOMICIDE OFFENDERS WITH SCHIZOPHRENIA
}

Katharina Nymo Engelstad ${ }^{\mathrm{a}, *}$, Anja Vaskinn ${ }^{\mathrm{b}, \mathrm{c}}$, Anne-Kari Torgalsbøen ${ }^{\mathrm{d}}$, Christine Mohn ${ }^{\mathrm{a}}$, Bjørn Lau ${ }^{d, e}$, Bjørn Rishovd Rund ${ }^{\text {a, d }}$

${ }^{\text {a}}$ Research Department, Vestre Viken Hospital Trust, P. O. Box 800, 3004 Drammen, Norway. E-mails: k.n.engelstad@psykologi.uio.no, h.c.mohn@psykologi.uio.no, b.r.rund@psykologi.uio.no

${ }^{b}$ NORMENT K. G. Jebsen Centre for Psychosis Research, Division of Mental Health and Addiction, Oslo University Hospital, P. O. Box 4956 Nydalen, 0424 Oslo, Norway. E-mail: anja.vaskinn@medisin.uio.no

'Institute of Clinical Medicine, Faculty of Medicine, University of Oslo, P. O. Box 1039

Blindern, 0315 Oslo, Norway.

${ }^{\mathrm{d}}$ Department of Psychology, Faculty of Social Sciences, University of Oslo, P. O. Box 1094

Blindern, 0317 Oslo, Norway. E-mails: a.k.torgalsboen@psykologi.uio.no,

bjorn.lau@psykologi.uio.no

${ }^{\mathrm{e}}$ Lovisenberg Diaconal Hospital, P. O. Box 4970 Nydalen, 0440 Oslo

*Corresponding author: Katharina Nymo Engelstad, Research Department, Vestre Viken Hospital Trust, P. O. Box 800, 3004 Drammen, e-mail: k.n.engelstad@ psykologi.uio.no, phone: +4798067682.

\begin{abstract}
Background: Our ability to predict and prevent homicides committed by individuals with schizophrenia is limited. Cognitive impairments are associated with poorer functional outcome in schizophrenia, possibly also homicide. The aim of the current study was to investigate global and specific cognition among homicide offenders with schizophrenia (HOS). Methods: Twenty-six HOS were compared to 28 individuals with schizophrenia and no history of violence (non-HOS), and a group of healthy controls (HC, $n=151)$. HOS and non-HOS participants were recruited from in- and outpatient units across Norway. An extensive neuropsychological test battery was administered. Results: HOS participants performed significantly weaker than $\mathrm{HC}$ in all cognitive domains. Further, statistically significant differences between HOS and non-HOS participants were found for IQ $(d=.52)$ and verbal learning $(d=.82)$, with larger impairments in the HOS compared to the non-HOS group. Conclusions: Our results indicate that HOS participants show clinically significant impairments in global and specific cognition.
\end{abstract}

Keywords: schizophrenia, homicide, violence, cognition, neuropsychology, verbal learning 


\section{Introduction}

\subsection{Schizophrenia, violence and homicide}

A diagnosis of schizophrenia is associated with an increased risk of committing homicide, it is estimated that individuals with this diagnosis commit $6 \%$ of all homicides [1]. The percentage is higher, probably about $20 \%$, in jurisdictions with low homicide rates such as the Scandinavian countries [2].

A meta-analysis of 110 studies investigating risk factors of violence in psychosis concluded that hostile behaviour, non-adherence to psychotherapy, recent drug and alcohol abuse, non-adherence to medication, as well as a history of criminal behaviour were the strongest predictors [3].

Although several risk factors for violence have been detected, current risk assessment tools are limited with regards to predicting violence in general [4], and specifically in schizophrenia [5]. Adding new factors that are of importance to violence in such risk assessment tools could aid violence prediction. Increased knowledge of the characteristics of individuals with a diagnosis of schizophrenia who commit severe violence is a step in the direction of improving current violence risk assessment tools.

\subsection{Is cognitive deficit a risk factor for violence in schizophrenia?}

Cognitive impairment is a defining feature of $[6,7]$ and associated with functioning $[8$, 9] in schizophrenia. Severe interpersonal violence, such as homicide, is an example of very poor functional outcome. From this follows that impaired cognition may be a predictor of homicide in individuals with schizophrenia. A proposition of lower general intelligence (IQ) as a potential risk factor of violence was made decades ago, although this was a study of violence in criminal offenders and not specifically individuals with schizophrenia [10].

Recent research has yielded optimism when it comes to the inclusion of cognitive factors in future violence risk assessment tools [11, 12]. A study of 25 homicide offenders with schizophrenia (HOS) [12] found impairments in a measure of general IQ. The researchers also found impairments in specific cognitive domains, including speed of processing, visuoconstructional abilities and a test of reasoning. A follow-up study of the same HOS included a control group of participants with schizophrenia who did not have a history of violence (non-HOS) [13]. The results indicated that HOS had relatively larger impairments than non-HOS in a measure general IQ, memory and executive functioning. IQ as a possible predictor of inpatient violence has also been suggested by Fullam and Dolan [14], who found lower IQ scores among violent than non-violent participants. In a recent 
review of the literature on violence and neuropsychological function in schizophrenia, Sedgwick, Young, Baumeister, Greer, Das and Kumari [15] concluded that there was evidence for an association between lower IQ, memory and executive function and violence in schizophrenia.

While some studies find a more pronounced generalized deficit in violent than in nonviolent schizophrenia, others have focused on specific cognitive domains. A few studies [1618], but not all [14], have found that poor executive function is associated with increased violence risk. Another study by Corbett, Karyadi, Kinney, Nitch, Bayan and Williams [19] found reduced verbal learning abilities among forensic inpatients with schizophrenia spectrum disorders, with scores about 1.5 standard deviations below the normative mean.

There are several limitations to the existing literature. First, as pointed out by Fullam and Dolan [14], definitions of violence are heterogeneous. While Stratton, Brook and Hanlon [12] focused solely on homicide, Brugman, Lobbestael, von Borries, Bulten, Cima, Schuhmann et al. [11] investigated verbal and physical violence collapsed. Second, we cannot draw inferences about violence risk in schizophrenia from studies of criminal offenders without mental illness $[10,16]$. Third, cognitive deficit as a risk factor for violence in schizophrenia has often been investigated with few tests [11, 19], although there are exceptions [13, 15, 17, 20]. O’Reilly, Donohoe, Coyle, O'Sullivan, Rowe, Losty et al. [20] applied the Measurement and Treatment Research to Improve Cognition in Schizophrenia (MATRICS) Consensus Cogntive Battery (MCCB) in a study of a forensic population with schizophrenia. They found that neurocognition and social cognition predicted inpatient violence over a 12 month follow-up period. Social cognition and neurocognition accounted for $34 \%$ of the variance in violence after controlling for age and gender. However, both those who committed violence during the follow-up period and those who did not had a history of violence prior to being admitted to the forensic hospital. Possibly, institutional violence could have other causes than non-institutional violence, suggesting that situational factors can be particularly important in institutional violence [21].

In the current study, some of the challenges presented above are addressed through an extensive investigation of cognition and homicide in schizophrenia. Specifically, we will explore cognitive characteristics among those who have a diagnosis of schizophrenia that have committed homicide. We ask if HOS participants show global cognitive impairments compared to non-HOS and healthy controls (HC). In addition, we will examine if HOS participants show specific cognitive impairments in the executive functioning and verbal learning domains. 


\section{Material and methods}

\subsection{Participants}

The HOS study is a cross-sectional comparative study. It was conducted at Vestre Viken Hospital Trust in Norway, in collaboration with a number of in- and outpatient units across the whole country. Data were collected by the first author between October 2015 and June 2017.

Participants were two groups of individuals with a diagnosis of schizophrenia or schizoaffective disorder (Table 1). Twenty-six of them were sentenced to compulsory mental care for homicide or homicide attempt (HOS group), and 28 had no history of interpersonal violence (non-HOS group). Diagnostic evaluations were made by clinicians at collaborating units prior to inclusion in the current study and were based on the International Statistical Classification of Diseases and Related Health Problems (ICD-10) [22]. Participants were excluded if they had insufficient knowledge of Norwegian language, i.e. were unable to undergo clinical interviews and cognitive testing in Norwegian. Norwegian language skills were evaluated qualitatively before the participant signed informed consent. One HOS who was eligible for participation was excluded due to insufficient comprehension of Norwegian.

At the time of inclusion, all participants received antipsychotic medication (Table 1). Medication Defined Daily Dose (DDD) was calculated for each participant according to World Health Organization (WHO) guidelines [23]. Information on participants' background, including illness history and violent episodes (HOS group), was available from medical records and participants' treating clinicians.

To maximize the number of eligible participants for the HOS group, patients were recruited regardless of time since the violent offense. Both participants who had committed homicide and homicide attempt were included in the HOS group, because it is often arbitrary or circumstantial whether a severely violent act towards another person is lethal or not.

Participants were initially informed of the study by their treating clinician, and received further information by the first author upon acceptance. All participants gave written informed consent prior to assessments. The study was approved by the Regional Committee for Medical and Health Research Ethics (REC South East 2015/713).

The healthy control group (HC) were 151 individuals who participated in the Norwegian MCCB standardization study [24]. Participants in the standardization study did not have a history of severe mental illness, substance abuse or severe head trauma. They also had sufficient knowledge of Norwegian language to undergo tests in Norwegian. For an 
extensive description of exclusion criteria of the Norwegian MCCB reference study, see Mohn, Sundet and Rund [24].

\subsection{Measures}

\subsubsection{Symptom measure.}

Symptoms were assessed with the Positive and Negative Syndrome Scale (PANSS) [25]. Symptom levels are shown in Table 1. Scores are based on a validated five factor PANSS model, and includes positive, negative, disorganized, excited and depressed symptoms $[26,27]$.

\subsubsection{Measures of cognition.}

Global cognition was assessed with the 2-subtest version of the Wechsler Abbreviated Scale of Intelligence (WASI) [28], consisting of the Vocabulary and Matrix Reasoning subtests. A composite score derived from the MCCB norms [29] also indexed global cognition.

Specific cognitive functioning was examined with a number of neuropsychological tests (Table 2). The MCCB [29] comprises seven cognitive domains assessed with ten different tests: Speed of processing; Trail Making Test - Part A (TMT-A), Brief Assessment of Cognition in Schizophrenia (BACS) Symbol Coding and Category Fluency: Animal Naming. Attention/vigilance; Continuous Performance Test - Identical Pairs. Working memory; Wechsler Memory Scale - third edition (WMS-III) Spatial Span and Letter-Number Span (LNS). Verbal learning; Hopkins Verbal Learning Test - Revised (HVLT-R). Visual learning; Brief Visuospatial Memory Test - Revised (BVMT-R). Reasoning/problem solving; Neuropsychological Assessment Battery (NAB): Mazes. Social cognition; Mayer-SaloveyCaruso Emotional Intelligence Test (MSCEIT): Managing Emotions. Standard scores were calculated for each domain based on age- and gender corrected US norms.

Two studies investigating the psychometric properties of the Norwegian translation of the MCCB showed that the translated version was sensitive to the cognitive deficits shown by patients with schizophrenia compared to healthy controls, in all domains covered by the test battery, except from the MSCEIT test [30, 31].

Because the MCCB does not extensively target executive functions, we added the Delis-Kaplan Executive Function System (D-KEFS) Color-Word Interference Test (CWIT) to our test battery [32]. The CWIT consists of four conditions where the first two conditions measure basic abilities, colour naming and reading colour names. The third and fourth 
conditions, inhibition and inhibition/switching, challenge executive abilities. A standard score for each condition derived from age corrected norms was used in the analyses.

\subsection{Statistics}

All statistical analyses were performed using The Statistical Package for the Social Sciences (IBM SPSS Statistics for Windows, version 24.0) [33].

Univariate analyses of variance (ANOVAs) were applied to examine differences in clinical, demographical and cognitive functioning between the groups. Only the HOS and non-HOS groups were compared on executive functioning as indexed by the CWIT. The HC group was added in analyses of global and specific cognition measured with WASI and MCCB. For analyses that included three groups and that yielded significant main effects, ANOVAS were followed by post hoc tests (Tukey) to infer which group differed from the others.

Alpha level was set at .05. We decided not to correct for multiple comparisons because of the small sample size in the HOS and non-HOS groups and the risk of committing type II errors.

Two different effect sizes are reported (see Table 2). Partial eta squared was used to describe the overall strength of group differences in the analyses including the three study groups. In addition, we calculated effect sizes for the differences between HOS and non-HOS groups (Cohen's $d$ ) using the pooled standard deviation (Table 2).

\section{Results}

\subsection{Global cognition}

We detected a significant effect of group membership on IQ level (Table 2). A followup post hoc analysis revealed that HOS participants had significantly lower IQ scores than both non-HOS and HC participants. The difference between non-HOS and HC was statistically significant. The three groups also differed significantly on another global measure of cognitive functioning, the MCCB composite score (Table 2, Figure 1). A post-hoc test indicated that the significant group difference was driven mainly by the discrepancy between HC and the two schizophrenia (SZ) groups. The non-HOS group performed better than HOS participants, although this difference did not reach statistical significance. 


\subsection{Specific cognitive functions}

Follow-up analyses yielded similar results: The groups differed significantly on all MCCB cognitive subdomains (Table 2, Figure 1). For all domains, except verbal learning, the significance was driven by HC outperforming both clinical groups. There were no significant differences between HOS and non-HOS groups. However, for the verbal learning domain, the difference between the SZ groups was significant. For this domain, HOS had lower scores than non-HOS.

Univariate analyses of variance for the four CWIT subtests measuring executive function (Table 2) revealed no significant differences between HOS and non-HOS for any condition.

\subsection{Follow-up analyses}

Because there were significantly more participants in the HOS than in the non-HOS group that were not native Norwegian speakers, we repeated the analyses that had yielded significant differences between the HOS and non-HOS group on only native speaking participants. HOS participants were still outperformed by the non-HOS group. The effect sizes for the IQ measure was medium sized, $(d=.52)$, but the difference was no longer statistically significant $\left(F_{(1,37)}=2.45, p=.13\right)$. For the verbal learning domain the group difference between HOS and non-HOS participants remained statistically significant $\left(F_{(1,37)}\right.$ $=5.25, p=.03)$, and the effect size was large $(d=.82)$.

\section{Discussion}

In this study, we compared two groups of participants with schizophrenia, one that had committed homicide or homicide attempt and one that had not committed interpersonal violence, to a group of healthy controls. Our aim was to examine the cognitive characteristics of HOS using a standardized, extensive, cognitive test battery. We asked if HOS participants showed global cognitive deficits, as well as specific deficits in the verbal learning and executive functioning domains.

\subsection{Global cognition}

First, we found large IQ differences between the groups, with mean IQ scores of 87, 98 and 109 for the HOS, non-HOS and HC respectively. Individuals with schizophrenia have, on average, lower IQ than non-affected peers, and low IQ may be an intrinsic risk factor for later development of schizophrenia [34]. However, HOS participants had, on average, an IQ 
score 12 points below non-HOS. The difference between HOS and non-HOS thereby approached one standard deviation, whereas the difference between HOS and HC amounted to more than 1.5 standard deviation. This is in line with the findings of Stratton, Brook and Hanlon [12] whose HOS participants had an average IQ of 79.

Attempts to disentangle mechanisms behind the lower IQ among HOS participants in the present study would be mere speculation, although some researchers have pointed to associations between IQ, violence, trauma and schizophrenia. Meta-analytic findings by Ttofi, Farrington, Piquero, Lösel, DeLisi and Murray [35] indicated that high intelligence was a possible protective factor against criminal offending. Another study by Oakley, Harris, Fahy, Murphy and Picchioni [36] pointed out that exposure to violence in childhood could be a causal factor for later violent offences in schizophrenia. Possibly, as hypothesized by Koenen, Moffitt, Caspi, Taylor and Purcell [37], childhood trauma could have an impact on neurodevelopment and thereby be a causal factor of lower IQ. Further research on these associations is needed to warrant relevance for the current sample.

A second finding was the significant differences on the MCCB cognitive composite score, with HC performing significantly better than HOS and non-HOS. Non-HOS participants performed better than HOS participants, but this difference did not reach statistical significance.

\subsection{The MCCB cognitive subdomains}

Exploration of the seven cognitive subdomains of the MCCB showed a consistent pattern of performance, with HC performing significantly better than both non-HOS and HOS participants in all domains. Non-HOS had higher mean scores than HOS in all cognitive domains, but the difference reached statistical significance only for verbal learning. Effect sizes for the difference between HOS and non-HOS were however approaching medium magnitude for the working memory, reasoning and social cognitive domains, while the effect size for the verbal learning domain was large.

HOS participants performed two standard deviations below HC in the verbal learning and social cognitive domains, while the difference was more than one standard deviation for the remaining domains. Social cognition was indexed by the MSCEIT test in this study, a test that has not been able to detect social cognitive deficits in all schizophrenia samples [31]. Future studies that investigate a broader array of social cognitive functions, using more sensitive tests, could inform us further of the nature of social cognitive impairments among HOS. 


\subsection{Executive function}

Exploration of executive functions among HOS and non-HOS using the CWIT revealed no significant differences between groups. Results for each of the four conditions were almost similar in both groups. Although previously found in some studies [16-18], we did not detect differences in executive functioning in our sample. However, neither Barkataki, Kumari, Das, Hill, Morris, O'Connell et al. [17] nor Enticott, Ogloff, Bradshaw and Fitzgerald [18] found differences between violent and non-violent offenders with schizophrenia on measures of inhibition (Stroop-like tasks). Meijers, Harte, Meynen and Cuijpers [16] did find such differences, but they did not investigate participants with schizophrenia. As suggested by Enticott, Ogloff, Bradshaw and Fitzgerald [18], these results could indicate that cognitive inhibition may be differently related to impulsivity in schizophrenia and non-schizophrenia populations.

\subsection{Clinical implications}

We found that HOS participants performed significantly weaker than both non-HOS and $\mathrm{HC}$ in the verbal learning domain, which was indexed by the HVLT - R. HOS performed about 1.5 standard deviations below the normative mean. This deviation is in the same range as a study by Corbett, Karyadi, Kinney, Nitch, Bayan and Williams [19], and the authors point to the potential negative consequences of verbal learning impairments, because most of the non-pharmacological treatments are based on talking. In addition, verbal learning was previously found to be one of the cognitive domains that is most consistently associated with different aspects of functional outcome, and "it appears that verbal memory and vigilance are critical prerequisite capacities for skill acquisition in psychosocial rehabilitation programs" (Green [38], p. 325). Our results could indicate that particular caution should be taken in treatment and rehabilitation of violent patients with schizophrenia to ensure that verbal learning impairments are not an obstacle for the patient to learn and understand information relevant for the rehabilitation process.

Previous studies such as Simonsen, Sundet, Vaskinn, Ueland, Romm, Hellvin et al. [39] have defined clinically significant impairments as scores 1.5 standard deviations below normative mean. Concerning the current study, the HOS group performed 1.5 standard deviation below normative mean on several measures. Following this approach, clinically significant impairments were found in both global cognition (MCCB composite), and several specific cognitive domains (speed of processing, working memory, verbal learning, attention/vigilance and social cognition). For the remaining results (IQ, visual learning and 
reasoning/problem solving), performance of the HOS group approached 1.5 standard deviations below normative mean. Although not all differences in cognitive functioning between non-HOS and HOS participants reached statistical significance, we believe the relatively large impairments shown by the HOS group compared to a normative mean have clinical implications. These deficits would be expected to have substantial functional consequences.

In this study we have investigated neuropsychological characteristics of HOS independent of other potentially important covariates such as substance abuse, trauma exposure, or other factors that could contribute to increased homicide risk in interaction with neuropsychological functioning. Large, Smith and Nielssen [1] also present the role of lower socio-economic status, and the effect of migration, and how this affects treatment access, as potential factors to investigate in association with homicide and schizophrenia. These topics are beyond the scope of the current study, but further examination in a larger HOS sample could contribute to improving violence risk assessment for this group.

\subsection{Strengths and limitations}

Strengths of the study include clearly defined groups when it comes to history of violence, as well as a healthy control group. The application of a comprehensive neuropsychological test battery developed specifically for schizophrenia is another advantage. It is estimated that 80 people are currently sentenced to compulsory mental care for homicide or homicide attempt in Norway [40]. We have collected information on $26 \mathrm{HOS}$, which equalizes as much as $32.5 \%$ of the relevant population. Participants were recruited from both rural and urban areas across the country, and we therefore believe our HOS group is representative of the HOS population in Norway.

Some limitations should however be taken into consideration. Although we have included a substantial percentage of the HOS population in Norway, our HOS sample remains quite small. The cross-sectional design means that there is a temporal gap between violent offense and inclusion in the study, for some of our variables of interest this time gap could have an impact on the results.

A general critique of studies investigating associations with violence is that we cannot rule out that our results are not specifically indicators of violence risk, but a more general tendency towards antisocial behaviour [4]. It is possible that weaker cognitive performance among HOS participants than non-HOS participants is not associated with HOS having committed violence but having shown criminal or anti-social tendencies in a broader sense. 
Significantly more HOS than non-HOS participants did not speak Norwegian as their native language, which may have influenced our results. We have, however, qualitatively evaluated the validity of each participant's test results. Additionally, we conducted ANOVAS comparing only HOS and non-HOS participants whose native language was Norwegian. The pattern of the results remained mainly the same. Based on the qualitative evaluations and follow-up analyses, we find it most likely that our results are not explained by differences in native language.

The inclusion of a measure of pre-morbid IQ would have given more substance to the interpretation of differing IQ scores in the HOS and non-HOS groups, particularly because of relatively long illness duration for both patient groups, as well as a time gap since their violent offense for the HOS group.

We found that HOS participants received significantly higher doses of antipsychotic medication than non-HOS (Table 1). This is not surprising, considering the treatment regimen for this group, where the control of psychotic symptoms is of primary importance. We cannot rule out the possibility that this has affected our results. However, we feel confident that, in this case, the higher antipsychotic medication dose is a reflection of the severity of the disorder which also shows itself in larger neuropsychological impairments. Also, our results align with some previous studies that did not find significant associations between medication and cognitive functioning $[41,42]$.

\section{Conclusions}

In this study we explored cognitive characteristics of HOS, assessing both global and specific cognition.

Main findings indicate that HOS had significantly lower IQ scores than the non-HOS group. Non-HOS also outperformed HOS on the MCCB, although the difference reached statistically significance only for the verbal learning domain. HC performed significantly better than both non-HOS and HOS on global cognition as well as in all MCCB cognitive subdomains.

\section{Role of the funding source}

The study was funded by Vestre Viken Hospital Trust, grant number 9603009. The funding source had no role in study design, data collection and analysis, decision to publish or preparation of the manuscript. 


\section{Declarations of interest}

None. 


\section{References}

[1] Large M, Smith G, Nielssen O. The relationship between the rate of homicide by those with schizophrenia and the overall homicide rate: a systematic review and metaanalysis. Schizophr Res. 2009;112(1-3):123-9.

[2] Gottlieb P, Gabrielsen G, Kramp P. Psychotic homicides in Copenhagen from 1959 to 1983. Acta Psychiatr Scand. 1987;76(3):285-92.

[3] Witt K, van Dorn R, Fazel S. Risk factors for violence in psychosis: systematic review and meta-regression analysis of 110 studies. PLoS One. 2013;8(2):e55942.

[4] Coid JW, Yang M, Ullrich S, Zhang T, Sizmur S, Farrington D, et al. Most items in structured risk assessment instruments do not predict violence. The Journal of Forensic Psychiatry \& Psychology. 2011;22(1):3-21.

[5] Singh JP, Serper M, Reinharth J, Fazel S. Structured assessment of violence risk in schizophrenia and other psychiatric disorders: a systematic review of the validity, reliability, and item content of 10 available instruments. Schizophr Bull. 2011;37(5):899-912.

[6] Kahn RS, Keefe RSE. Schizophrenia is a cognitive illness: Time for a change in focus. JAMA Psychiatry. 2013;70(10):1107-12.

[7] Rund BR, Melle I, Friis S, Larsen TK, Midboe LJ, Opjordsmoen S, et al. Neurocognitive dysfunction in first-episode psychosis: correlates with symptoms, premorbid adjustment, and duration of untreated psychosis. Am J Psychiatry. 2004;161(3):466-72.

[8] Bowie CR, Depp C, McGrath JA, Wolyniec P, Mausbach BT, Thornquist MH, et al. Prediction of real-world functional disability in chronic mental disorders: a comparison of schizophrenia and bipolar disorder. Am J Psychiatry. 2010;167(9):1116-24.

[9] Fu S, Czajkowski N, Rund BR, Torgalsboen AK. The relationship between level of cognitive impairments and functional outcome trajectories in first-episode schizophrenia. Schizophr Res. 2017;190:144-9.

[10] Robertson G, Taylor PJ, Gunn JC. Does violence have cognitive correlates? Br J Psychiatry. 1987;151:63-8.

[11] Brugman S, Lobbestael J, von Borries AK, Bulten BE, Cima M, Schuhmann T, et al. Cognitive predictors of violent incidents in forensic psychiatric inpatients. Psychiatry Res. 2016;237:229-37. 
[12] Stratton J, Brook M, Hanlon RE. Murder and psychosis: Neuropsychological profiles of homicide offenders with schizophrenia. Crim Behav Ment Health. 2016.

[13] Stratton J, Cobia DJ, Reilly J, Brook M, Hanlon RE. Differences in Neuropsychological Functioning Between Homicidal and Nonviolent Schizophrenia Samples. J Forensic Sci.n/a-n/a.

[14] Fullam RS, Dolan MC. Executive function and in-patient violence in forensic patients with schizophrenia. Br J Psychiatry. 2008;193(3):247-53.

[15] Sedgwick O, Young S, Baumeister D, Greer B, Das M, Kumari V. Neuropsychology and emotion processing in violent individuals with antisocial personality disorder or schizophrenia: The same or different? A systematic review and meta-analysis. Aust N Z J Psychiatry. 2017:4867417731525.

[16] Meijers J, Harte JM, Meynen G, Cuijpers P. Differences in executive functioning between violent and non-violent offenders. Psychol Med. 2017;47(10):1784-93.

[17] Barkataki I, Kumari V, Das M, Hill M, Morris R, O'Connell P, et al. A neuropsychological investigation into violence and mental illness. Schizophr Res. 2005;74(1):1-13.

[18] Enticott PG, Ogloff JR, Bradshaw JL, Fitzgerald PB. Cognitive inhibitory control and self-reported impulsivity among violent offenders with schizophrenia. J Clin Exp Neuropsychol. 2008;30(2):157-62.

[19] Corbett L, Karyadi KA, Kinney D, Nitch SR, Bayan SM, Williams M. Impaired verbal learning in forensic inpatients with Schizophrenia Spectrum Disorder. Applied Neuropsychology: Adult. 2017:1-8.

[20] O’Reilly K, Donohoe G, Coyle C, O’Sullivan D, Rowe A, Losty M, et al. Prospective cohort study of the relationship between neuro-cognition, social cognition and violence in forensic patients with schizophrenia and schizoaffective disorder. BMC Psychiatry. 2015;15:155.

[21] Gadon L, Johnstone L, Cooke D. Situational variables and institutional violence: a systematic review of the literature. Clin Psychol Rev. 2006;26(5):515-34.

[22] World Health Organization. ICD-10 : International statistical classification of diseases and related health problems. Geneva: World Health Organization; 2004.

[23] WHO Collaborating Centre for Drug Statistics Methodology. Guidelines for ATC classification and DDD assignment 2018 Oslo, Norway: WHO Collaborating Centre for Drug Statistics Methodology; 2017 [updated 15 February 2018. Available from: https://www.whocc.no/atc_ddd_index/. 
[24] Mohn C, Sundet K, Rund BR. The Norwegian standardization of the MATRICS (Measurement and Treatment Research to Improve Cognition in Schizophrenia) Consensus Cognitive Battery. J Clin Exp Neuropsychol. 2012;34(6):667-77.

[25] Kay SR, Fiszbein A, Opler LA. The positive and negative syndrome scale (PANSS) for schizophrenia. Schizophr Bull. 1987;13(2):261-76.

[26] Wallwork RS, Fortgang R, Hashimoto R, Weinberger DR, Dickinson D. Searching for a consensus five-factor model of the Positive and Negative Syndrome Scale for schizophrenia. Schizophr Res. 2012;137(1-3):246-50.

[27] Langeveld J, Andreassen OA, Auestad B, Faerden A, Hauge LJ, Joa I, et al. Is there an optimal factor structure of the Positive and Negative Syndrome Scale in patients with first-episode psychosis? Scand J Psychol. 2013;54(2):160-5.

[28] Wechsler D. Wechsler Abbreviated Scale of Intelligence (WASI). Norwegian manual supplement. Stockholm, Sweden: Pearson Assessment; 2007.

[29] Nuechterlein KH, Green MF. MATRICS consensus cognitive battery. Manual. Los Angeles, CA: MATRICS Assessment Inc.; 2006.

[30] Lystad JU, Falkum E, Mohn C, Haaland VO, Bull H, Evensen S, et al. The MATRICS Consensus Cognitive Battery (MCCB): performance and functional correlates. Psychiatry Res. 2014;220(3):1094-101.

[31] Holmén A, Juuhl-Langseth M, Thormodsen R, Melle I, Rund BR. Neuropsychological profile in early-onset schizophrenia-spectrum disorders: measured with the MATRICS battery. Schizophr Bull. 2010;36(4):852-9.

[32] Delis DC, Kaplan E, Kramer JH. The Delis-Kaplan Executive Function System. DKEFS. Norwegian Manual. Stockholm: Pearson Assessment; 2005.

[33] IBM Corp. IBM SPSS Statistics for Windows, Version 24.0. Armonk, NY: IBM Corp.; 2016.

[34] Reichenberg A, Weiser M, Caspi A, Knobler HY, Lubin G, Harvey PD, et al. Premorbid intellectual functioning and risk of schizophrenia and spectrum disorders. J Clin Exp Neuropsychol. 2006;28(2):193-207.

[35] Ttofi MM, Farrington DP, Piquero AR, Lösel F, DeLisi M, Murray J. Intelligence as a protective factor against offending: A meta-analytic review of prospective longitudinal studies. Journal of Criminal Justice. 2016;45(Supplement C):4-18.

[36] Oakley C, Harris S, Fahy T, Murphy D, Picchioni M. Childhood adversity and conduct disorder: A developmental pathway to violence in schizophrenia. Schizophr Res. 2016. 
[37] Koenen KC, Moffitt TE, Caspi A, Taylor A, Purcell S. Domestic violence is associated with environmental suppression of IQ in young children. Dev Psychopathol. 2003;15(2):297-311.

[38] Green MF. What are the functional consequences of neurocognitive deficits in schizophrenia? Am J Psychiatry. 1996;153(3):321-30.

[39] Simonsen C, Sundet K, Vaskinn A, Ueland T, Romm KL, Hellvin T, et al. Psychosocial function in schizophrenia and bipolar disorder: Relationship to neurocognition and clinical symptoms. J Int Neuropsychol Soc. 2010;16(5):771-83.

[40] NOU 2014:10. Skyldevne, sakkyndighet og samfunnsvern. 2014.

[41] Torgalsbøen A-K, Mohn C, Czajkowski N, Rund BR. Relationship between neurocognition and functional recovery in first-episode schizophrenia: Results from the second year of the Oslo multi-follow-up study. Psychiatry Res. 2015;227(2):185-91.

[42] Ekerholm M, Firus Waltersson S, Fagerberg T, Söderman E, Terenius L, Agartz I, et al. Neurocognitive function in long-term treated schizophrenia: A five-year follow-up study. Psychiatry Res. 2012;200(2):144-52. 


\section{Tables}

Table 1: Demography and clinical data.

\begin{tabular}{llll}
\hline & HOS, $\mathrm{n}=26$ & Non-HOS, $\mathrm{n}=28$ & HC, $\mathrm{n}=151$ \\
\hline Age & $38.2(7.3)$ & $36.7(10.1)$ & $34.1(8.9)$ \\
Sex, male $^{* *}$ & $25(96 \%)$ & $25(89 \%)$ & $76(50 \%)$ \\
Education, years $^{* *}$ & $9.6(2.2)$ & $11.1(1.6)$ & $12.9(2.5)$ \\
Diagnosis & 23 schizophrenia & 25 schizophrenia & - \\
& 3 schizoaffective & 1 schizoaffective & \\
Illness duration, years & $15.7(6.7)$ & $13.7(10.1)^{\mathrm{a}}$ & - \\
Medication, DDD ${ }^{*}$ b & $1.84(.80)$ & $1.36(.64)$ & - \\
Time since offense, years & 6.5 & - & - \\
Norwegian native language & $15(57.7 \%)$ & $24(85.7 \%)$ & - \\
Inpatients & $16(61.5 \%)$ & $3(10.7 \%)$ & - \\
PANSS positive (min-max 4-28) & $7.2(4.1)$ & $7.5(4.7)$ & - \\
PANSS negative (min-max 6-42) & $10.2(5.2)$ & $8.1(2.8)$ & - \\
PANSS disorganized (min-max 3-21) & $5.7(2.0)$ & $4.8(1.7)$ & - \\
PANSS excited (min-max 4-28) & $5.1(1.5)$ & $4.2(.5)$ & $7.0(2.8)$ \\
PANSS depressed (min-max 3-21) & $6.3(3.6)$ & & - \\
\hline
\end{tabular}

${ }^{*} p<.05 .{ }^{* *} p<.01{ }^{a} N=27 .{ }^{\mathrm{b}} \mathrm{DDD}=$ Defined Daily Dose [23] 
Table 2: Cognition among HOS, non-HOS and HC, results of ANOVAs.

\begin{tabular}{|c|c|c|c|c|c|c|}
\hline & $\begin{array}{l}\text { HOS } \\
(n=26)\end{array}$ & $\begin{array}{l}\text { Non-HOS } \\
(\mathrm{n}=28)\end{array}$ & $\begin{array}{l}\mathrm{HC} \\
(\mathrm{n}=151)\end{array}$ & Statistic & $\begin{array}{l}\text { Partial Eta } \\
\text { Squared }^{\mathrm{a}}\end{array}$ & $\begin{array}{l}\text { Cohen's } \\
d^{\mathrm{b}}\end{array}$ \\
\hline WASI IQ $^{c}$ & $87.0(16.7)$ & $98.0(15.8)$ & $108.5(14.3)^{\mathrm{d}}$ & $\begin{array}{l}F_{(2,198)}=26.2 \\
p<.01\end{array}$ & .21 & .68 \\
\hline $\begin{array}{l}\text { WASI } \\
\text { Vocabulary }\end{array}$ & $38.3(12.4)$ & $45.9(12.1)$ & $50.8(8.9)$ & $\begin{array}{l}F_{(2,200)}=18.9 \\
p<.01\end{array}$ & .16 & .62 \\
\hline $\begin{array}{l}\text { WASI Matrix } \\
\text { Reasoning }\end{array}$ & $43.8(12.6)$ & $50.4(13.2)$ & $57.6(7.7)$ & $\begin{array}{l}F_{(2,199)}=27.9 \\
p<.01\end{array}$ & .22 & .51 \\
\hline MCCB comp ${ }^{e}$ & $27.4(8.2)$ & $32.0(9.3)$ & $47.1(8.3)$ & $\begin{array}{l}F_{(2,202)}=85.8 \\
\mathrm{p}<.01\end{array}$ & .46 & .52 \\
\hline MCCB domains ${ }^{\mathrm{e}}$ & & & & & & \\
\hline Speed of processing & $29.9(9.2)$ & $32.2(9.3)$ & $49.0(9.7)$ & $\begin{array}{l}F_{(2,202)}=69.5 \\
p<.01\end{array}$ & .41 & .25 \\
\hline Working memory & $33.5(10.4)$ & $37.3(11.8)$ & $44.5(8.1)$ & $\begin{array}{l}F_{(2,202)}=21.0 \\
p<.01\end{array}$ & .17 & .34 \\
\hline Verbal learning & $34.5(4.2)$ & $40.5(10.5)$ & $47.3(9.3)$ & $\begin{array}{l}F_{(2,202)}=26.1 \\
p<.01\end{array}$ & .21 & .75 \\
\hline Visual learning & $37.2(13.2)$ & $39.3(13.9)$ & $51.4(8.6)$ & $\begin{array}{l}F_{(2,202)}=33.6 \\
p<.01\end{array}$ & .25 & .15 \\
\hline $\begin{array}{l}\text { Attention/ } \\
\text { vigilance }\end{array}$ & $32.5(10.0)$ & $33.1(8.2)$ & $45.5(8.0)$ & $\begin{array}{l}F_{(2,202)}=46.5 \\
p<.01\end{array}$ & .32 & .07 \\
\hline $\begin{array}{l}\text { Reasoning/ probl. } \\
\text { solv. }\end{array}$ & $37.9(8.0)$ & $41.2(8.6)$ & $49.6(9.5)$ & $\begin{array}{l}F_{(2,202)}=23.9 \\
p<.01\end{array}$ & .19 & .40 \\
\hline Social cognition & $33.3(12.2)$ & $37.9(11.1)$ & $49.6(10.3)$ & $\begin{array}{l}F_{(2,202)}=35.3 \\
p<.01\end{array}$ & .26 & .39 \\
\hline \multicolumn{7}{|l|}{$\mathrm{CWIT}^{\mathrm{f}}$} \\
\hline Colour naming & $5.3(3.3)$ & $5.7(3.4)$ & - & $\begin{array}{l}F_{(1,52)}=.17 \\
p=.69\end{array}$ & .003 & .12 \\
\hline Word reading & $7.7(2.7)$ & $8.5(5.0)$ & - & $\begin{array}{l}F_{(1,52)}=.62 \\
p=.44\end{array}$ & .01 & .20 \\
\hline Inhibition & $6.7(3.6)$ & $7.0(3.8)$ & - & $\begin{array}{l}F_{(1,52)}=.14 \\
p=.71\end{array}$ & .003 & .08 \\
\hline Inhibition/switching & $7.2(3.2)$ & $7.9(3.4)$ & - & $\begin{array}{l}F_{(1,52)}=.61 \\
p=.44\end{array}$ & .01 & .21 \\
\hline
\end{tabular}

${ }^{a}$ Partial eta squared for three groups. ${ }^{b}$ Cohen's $d$ for HOS and non-HOS groups. ${ }^{c} I Q$ scores: $M=100, S D=15$. ${ }^{d} N=147 .{ }^{e} T$-scores: $M=50, S D=10 .{ }^{f}$ Scaled scores: $M=10, S D=3$. 


\section{Figure legends}

Figure 1: MCCB results across cognitive domains. Average T-scores. $M=50, S D=10$.

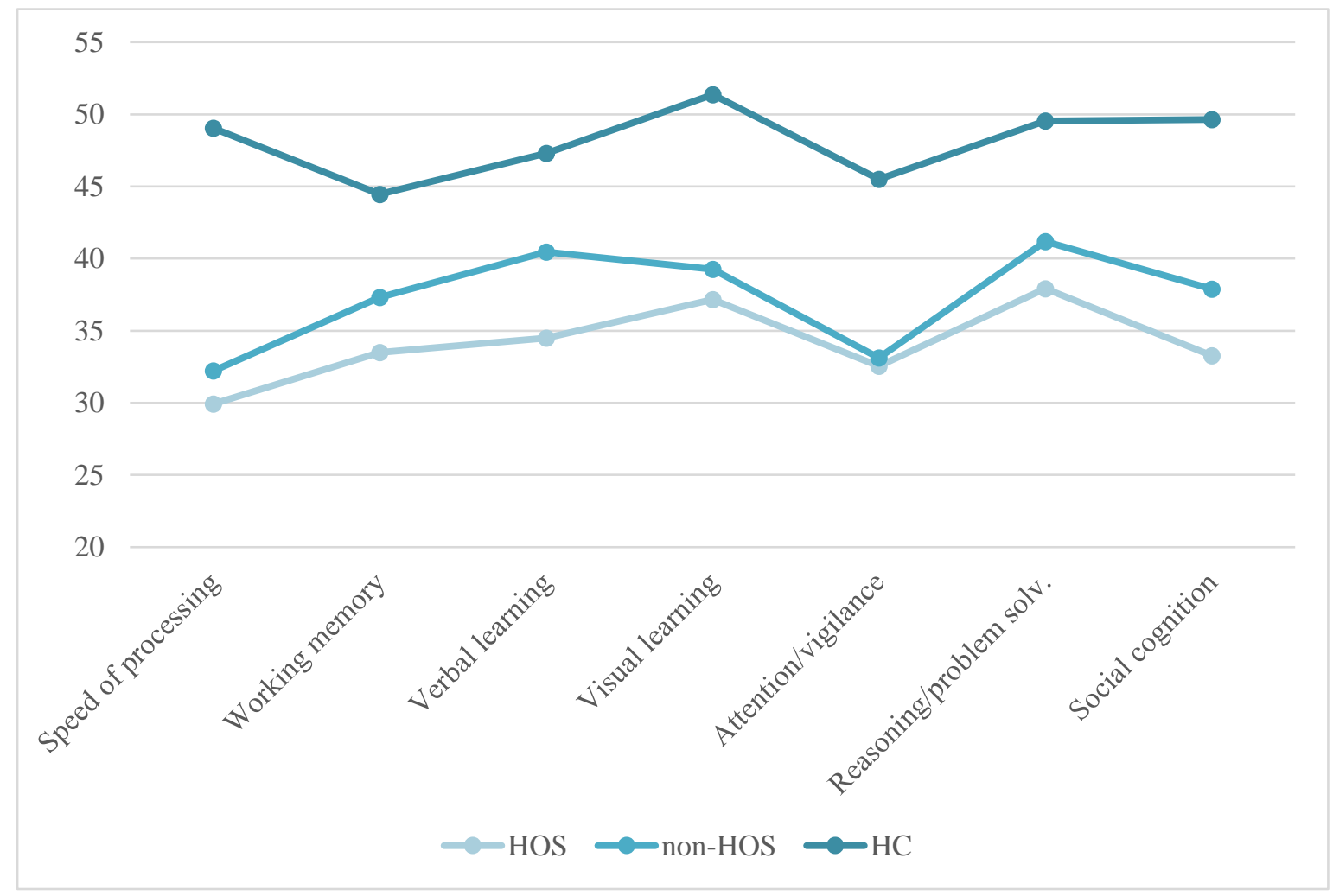

